\title{
Noether theorem of a kind of singular integral equation with Hilbert kernel on open arcs
}

\author{
Li-xia Cao ${ }^{1, a}$ \\ ${ }^{1}$ Mathematics College, Northeast Petroleum University, Daqing, Heilongjiang 163318, China \\ acaolixia98237@163.com
}

Keywords: Hilbert kernel; singular integral equation; general solutions; solvable conditions

\begin{abstract}
We discussed a kind of singular integral equation with Hilbert kernel on open arcs lying in a period strip. By using the method of complex functions, we obtained the extended Plemelj Formula with Hilbert kernel, and based on this, we obtained the general solutions and the solvable conditions for this kind of characteristic singular integral equation with Hilbert kernel on open arcs.
\end{abstract}

\section{Introduction}

In $[1,2,3,4,5]$, the authors discussed the singular integral equation with Cauchy kernel on the real half-line, on the real line, or on a complicated contour. In [6], the authors discussed Numerical solution of a singular integral equation with Cauchy kernel in the plane contact problem. While the solvable Noether theorem for the singular integral equations with Hilbert kernel on open arcs, has few been discussed. Here we study a kind of singular integral equation with Hilbert kernel on open arcs by transforming it into the periodic Riemann boundary value problem, then give the general solutions and the solvable conditions.

\section{The characteristic singular integral equation with Hilbert kernel on open arcs}

Suppose that $L=\sum_{j} L_{j}$ is a finite set of nonintersecting open arcs, with period $a \pi$ lying entirely in the in the same periodic region $S:|\operatorname{Re} z|<a \pi / 2$, and being positively oriented. We assume that $A(t), B(t), f(t) \in H(L)$ are the given functions. We consider the following the characteristic singular integral equation with Hilbert kernel for a Hölder continuous function $\varphi(t)$ on $L$

$$
K^{0} \varphi \equiv A\left(t_{0}\right) \varphi\left(t_{0}\right)+\frac{B\left(t_{0}\right)}{a \pi i} \int_{L} \cot \frac{t-t_{0}}{a} \varphi(t) d t, \quad t_{0} \in L,
$$

We shall assume, without restricting the general case, that $A^{2}(t)-B^{2}(t) \neq 0$. And suppose $\mathrm{t}$ hat the different points $C_{1}, C_{2}, \cdots, c_{n}$ are the all nodes on $L$ (including the end-points of $L_{j}$ and the discontinuity points of $A(t), B(t)$ and $f(t))$. Without loss of generality, no special condition is needed except for the requirement that $\varphi \in h=h\left(c_{1}, c_{2}, \cdots, c_{q}\right)(q \leq n)$, that is $\varphi$ is bounded $\mathrm{n}$ ear $c_{1}, c_{2}, \cdots$ and $c_{q}$, while $\varphi$ has a singularity of order less than 1 at any other nodes. For $L$, we permit it to arrive at the boundary of the region $S$, and in this case we regarded $c$ and $c \pm a \pi$ as the same one.

\section{Transformation of given equation}

Lemma 1 (Plemelj Formula with Hilbert kernel) Suppose that $L$ is a close or open smooth curve. If $g(t) \in H$ with period $a \pi(a>0)$, and $\Psi(z)=\frac{1}{2 a \pi i} \int_{L} g(t) \cot \frac{t-z}{a} \mathrm{~d} t, z \notin L, \quad Z \notin L$. Then

$$
\Psi^{ \pm}\left(t_{0}\right)= \pm \frac{1}{2} g\left(t_{0}\right)+\frac{1}{2 a \pi i} \int_{L} g(t) \cot \frac{t-t_{0}}{a} d t, \quad t_{0} \in L,
$$

Proof. With the aid of the Laurent series expansion of $\cot [(\tau-t) / a]$, we can obtain the Lemma by using similar method in [1]. Let 


$$
\begin{gathered}
S(t)=A(t)+B(t), \quad D(t)=A(t)-B(t), \\
\kappa_{j}=\frac{1}{2 \pi i}\left[\log \frac{A(t)-B(t)}{A(t)+B(t)}\right]_{L_{j}}=\frac{1}{2 \pi}\left[\arg \frac{D}{S}\right]_{L_{j}} .
\end{gathered}
$$

$\kappa=\sum_{j} \kappa_{j}$ is called the index of Eq. (2).

The canonical function of Eq. (2) in class $h=h\left(c_{1}, c_{2}, \cdots, c_{q}\right)$ can be given in the form

$$
X(z)=I I(z) e^{\Gamma(z)},
$$

where

$$
\begin{gathered}
I I(z)=\prod_{j=1}^{n}\left(\tan \frac{z}{a}-\tan \frac{C_{j}}{a}\right)^{\kappa_{j}}, \\
\Gamma(z)=\frac{1}{2 a \pi i} \int_{L} \log G(t) \cot \frac{t-z}{a} d t, \\
G(t)=[A(t)-B(t)] /[A(t)+B(t)],
\end{gathered}
$$

where $\log G(t)$ is a definite arbitrary continuous branch on $L_{j}$. Denote

$$
G_{\infty}=X(-\infty i) / X(+\infty i) \text {. }
$$

Through calculation, we obtain

$$
G_{\infty}=(-1)^{\kappa} e^{-i v}=e^{-i \mu \pi}
$$

where

$$
\left.\begin{array}{l}
v=\sum_{j=1}^{p} \frac{1}{a \pi i} \int_{L_{j}} \log \frac{G(t) d t}{\left(\tan \frac{t}{a}-\tan \frac{Z_{j}}{a}\right)^{\kappa_{j}}}-\frac{2}{a} \sum_{j=1}^{p} \kappa_{j} Z_{j}, \\
\mu=\frac{v}{\pi}-\kappa .
\end{array}\right\}
$$

Let

$$
\Phi(z)=\frac{1}{2 a \pi i} \int_{L} \varphi(t) \cot \frac{t-z}{a} d t,
$$

then $\Phi(z)$ is a sectionally holomorphic function with period $a \pi$, and with jump $L$. From the extended Plemelj formula with Hilbert kernel (2), we have

$$
\varphi\left(t_{0}\right)=\Phi^{+}\left(t_{0}\right)-\Phi^{-}\left(t_{0}\right), t_{0} \in L
$$

Eq.(2) can be transformed into the PR problem (period Riemann boundary problem)

$$
\Phi^{+}\left(t_{0}\right)=G\left(t_{0}\right) \Phi^{-}\left(t_{0}\right)+g\left(t_{0}\right), t_{0} \in L,
$$

where

$$
g\left(t_{0}\right)=f\left(t_{0}\right) /\left[A\left(t_{0}\right)+B\left(t_{0}\right)\right] .
$$

By noting that $\lim _{z \rightarrow \pm \infty i} \cot \frac{t-z}{a}= \pm i$, we conclude that $\Phi( \pm \infty i)= \pm \frac{1}{2 a \pi} \int_{L} \varphi(t) \mathrm{d} t($ finite $)$, so we have

$$
\Phi(-\infty i)=-\Phi(+\infty i)
$$

And conversely, if $\Phi(z)$ is a sectionally holomorphic function with period $a \pi$, and with jump $L$, then $\Phi(z)$ must satisfy the boundary condition (13) and the additive condition (15), so the function $\varphi\left(t_{0}\right)$ determined by (12) is actually the solution of Eq.(1). Therefore, we obtain the following theorem

Theorem 3.1 Eq.(1) is equivalent to the PR problem (13) with additive (15), moreover solution of Eq.(1) is given by (12) when the PR problem (13) is solved.

\section{The solving of the characteristic singular integral equation on open arcs}

Let 


$$
Z(t)=[A(t)+B(t)] X^{+}(t)=[A(t)-B(t)] X^{-}(t),
$$

and solve Eq.(1) in class $h=h\left(c_{1}, c_{2}, \cdots, c_{q}\right)$, we obtain by calculation and arrangement that $1^{\circ}$ when $\kappa>0$, the general solution Eq.(1) is

$$
\varphi\left(t_{0}\right)=A^{*}\left(t_{0}\right) f\left(t_{0}\right)-\frac{B^{*}\left(t_{0}\right) Z\left(t_{0}\right)}{a \pi i} \int_{L} \frac{f(t)}{Z(t)} \cot \frac{t-t_{0}}{a} d t+B^{*}\left(t_{0}\right) Z\left(t_{0}\right) P_{\kappa}\left(\tan \frac{t_{0}}{a}\right),
$$

where the coefficients of $P_{\kappa}$ satisfy

$$
\begin{gathered}
-\left(c_{0}-c_{2}+\cdots\right) \cos \frac{v}{2}+\left(c_{1}-c_{3}+\cdots\right) \sin \frac{v}{2}=f_{0} \sin \frac{v}{2} \\
f_{0}=\frac{1}{a \pi d} \int_{L} \frac{f(t)}{Z(t)} d t \\
A^{*}(t)=A(t) /\left[A^{2}(t)-B^{2}(t)\right], B^{*}(t)=B(t) /\left[A^{2}(t)-B^{2}(t)\right],
\end{gathered}
$$

$2^{\circ}$ when $\kappa=0$, there are two cases:

if $v \neq(2 k+1) \pi$, Eq.(1) has unique solution

$$
\varphi\left(t_{0}\right)=A^{*}\left(t_{0}\right) f\left(t_{0}\right)-\frac{B^{*}\left(t_{0}\right) Z\left(t_{0}\right)}{a \pi i} \int_{L} \frac{f(t)}{Z(t)} \cot \frac{t-t_{0}}{a} d t-f_{0} \tan \frac{v}{2},
$$

and if $v=(2 k+1) \pi$, Eq.(1) has unique solution

$$
\varphi\left(t_{0}\right)=A^{*}\left(t_{0}\right) f\left(t_{0}\right)-\frac{B^{*}\left(t_{0}\right) Z\left(t_{0}\right)}{a \pi i} \int_{L} \frac{f(t)}{Z(t)} \cot \frac{t-t_{0}}{a} d t+c \cdot B^{*}\left(t_{0}\right) Z\left(t_{0}\right),
$$

$3^{\circ}$ when $\kappa<0$, Eq.(1) has unique solution

$$
\varphi\left(t_{0}\right)=A^{*}\left(t_{0}\right) f\left(t_{0}\right)-\frac{B^{*}\left(t_{0}\right) Z\left(t_{0}\right)}{a \pi i} \int_{L} \frac{f(t)}{Z(t)}\left(\cot \frac{t-t_{0}}{a}-\tan \frac{t}{a}\right) d t,
$$

if and only if the solvable condition(when $\kappa=-1$, the solvable condition is not required )

and the additive condition

$$
\int_{L} \frac{f(t)}{Z(t)}\left(\tan ^{j-1} \frac{t}{a}+\tan ^{j+1} \frac{t}{a}\right) d t=0 \quad, j=1, \cdots,-\kappa-1,
$$

$$
\int_{L} \frac{f(t)}{Z(t)} \sin \left(\frac{t}{a}-\frac{v}{2}\right) /\left(\cos \frac{t}{a}\right) d t=0
$$

Combining (24) and (25), we obtain the following solvable condition

$$
\left\{\begin{array}{l}
\int_{L} \frac{f(t)}{Z(t)}\left(\tan ^{j-1} \frac{t}{a}+\tan ^{j+1} \frac{t}{a}\right) d t=0, j=1, \cdots,-\kappa-1, \\
\int_{L} \frac{f(t)}{Z(t)}\left(\sin \frac{v}{2}-\cos \frac{v}{2} \tan \frac{t}{a}\right) d t=0 .
\end{array}\right.
$$

\section{The solving of the associative equation on open arcs}

The associative homogeneous of Eq.(1) is

$$
K^{\circ} \psi \equiv A\left(t_{0}\right) \psi\left(t_{0}\right)-\frac{1}{a \pi i} \int_{L} B(t) \psi(t) \cot \frac{t-t_{0}}{a} d t=0, t_{0} \in L,
$$

and we say that $h^{\prime}=h^{\prime}\left(c_{q+1}, c_{q+2}, \cdots, c_{n}\right)$ is associative class of $h=h\left(c_{1}, c_{2}, \cdots, c_{q}\right)$.It is clearly that the index of Eq.(27) is $\kappa^{\prime}=-\kappa$.

Similar to the solving of the characteristic singular integral equation on open arc, we obtain that when $\kappa^{\prime}>0$, Eq.(27) has the general solution in class $h^{\prime}$

$$
P_{\kappa^{\prime}}\left(\tan \frac{t_{0}}{a}\right) / Z\left(t_{0}\right)
$$

where $P_{\kappa^{\prime}}(w)=b_{0}+b_{1} w+\cdots+b_{\kappa^{\prime}} w^{\kappa^{\prime}}$ is an arbitrary polynomial of degree $\kappa^{\prime}$, and its coefficients satisfies the additive condition: 


$$
-\left(b_{0}-b_{2}+\cdots\right) \cos \frac{v}{2}+\left(b_{1}-b_{3}+\cdots\right) \sin \frac{v}{2}=0,
$$

Therefore we can take a set of linearly independent solutions of Eq.(27) as follows

$$
\begin{gathered}
\left(\sin \frac{v}{2} \tan ^{0} \frac{t}{a}-\cos \frac{v}{2} \tan ^{1} \frac{t}{a}\right) / Z(t),\left(\tan ^{0} \frac{t}{a}+\tan ^{2} \frac{t}{a}\right) / Z(t),\left(\tan ^{1} \frac{t}{a}+\tan ^{3} \frac{t}{a}\right) / Z(t), \\
\left(\tan ^{2} \frac{t}{a}+\tan ^{4} \frac{t}{a}\right) / Z(t), \cdots,\left(\tan ^{\kappa^{\prime}-3} \frac{t}{a}+\tan ^{\kappa^{\prime}-1} \frac{t}{a}\right) / Z(t),\left(\tan ^{\kappa^{\prime}-2} \frac{t}{a}+\tan ^{\kappa^{\prime}} \frac{t}{a}\right) / Z(t) .
\end{gathered}
$$

\section{Noether theorem of characteristic singular integral equation with Hilbert kernel on open arcs}

By comparing the results in 4 and 5, we can establish the following results.

Theorem 6.1 (Noether Theorem)

(a) The number of the linearly independent solutions of $K^{0} \varphi=0$ in class $h=h\left(c_{1}, c_{2}, \cdots, c_{q}\right)$ and $K^{\circ} \psi=0$ in class $h^{\prime}$ are all finite, denoted by $l$ and $l^{\prime}$ respectively. Moreover $l-l^{\prime}=\kappa$, where $\kappa$ is the index of operator $K^{0}$.

(b) $K^{0} \varphi=f$ is solvable in class $h=h\left(c_{1}, c_{2}, \cdots, c_{q}\right)$ iff the conditions

$$
\int_{L} \psi_{k}(t) f(t) d t=0, \quad k=0,1, \cdots, l^{\prime}
$$

are satisfied, where $\left\{\psi_{k}\right\}_{1}^{l^{\prime}}$ is the set of linearly independent solutions in class $h^{\prime}$ of $K^{\circ} \psi=0$.

\section{References}

[1] Muskhelishvili N I., Singular Integral Equations, Nauka, Moscow, 1968.

[2] D. Pylak; R. Smarzewski; M. A. Sheshko, A Singular Integral Equation with a Cauchy Kernel on the Real Half-Line, J. Differential Equations, Volume 41(2005) 1775-1788.

[3]B. G. Gabdulkhaevt; I. N. Tikhonov, Methods for Solving a Singular Integral Equation with Cauchy Kernel on the Real Line,J. Differential equations, Volume 44(2008) 980-990.

[4] M. A. Sheshko; S. M. Sheshko, Singular integral equation with Cauchy kernel on the real axis, J. Differential Equations, Volume 46(2010) 568-585.

[5] M. A. Sheshko; S. M. Sheshko, Singular integral equation with Cauchy kernel on a complicated contour, Differential Equations, Volume 47(,2011) 1344-1356.

[6] M. R. Capobianco; G. Criscuolo, Numerical solution of a singular integral equation with Cauchy kernel in the plane contact problem,J. Quarterly of Applied Mathematics, Volume 69(2011) 79-89. 